\title{
Taste and flavour: their importance in food choice and acceptance
}

\author{
Jane E. Clark \\ Product Perceptions Ltd., St George's House, Yattendon Road, Horley, Surrey RH6 7BS, UK
}

The present paper takes a look at the role of taste and flavour in consumers' food choice and acceptance. Are these sensory attributes the key to successful product development, or should food manufacturers be trying harder to understand what makes consumers tick?

\section{The enjoyment of food (or the 'feel good' factor)}

There are many reasons why we consume food, the obvious and most important one being to obtain nutrition for a healthy and happy body. However, in a society where food supply, safety and nutrition are more than adequately provided for by most major manufacturers, other issues relating to the consumers' food choice have grown in importance.

One aspect of food consumption, and one that sadly is often over-looked, is that consumers want to enjoy their food. In fact, many foods are consumed almost entirely for the pleasure value they impart. Products such as coffee, chocolate, ice cream and of course alcohol are often consumed to enhance positive states of mind, or at least to reduce the effects of the negative ones.

Ever increasing consumer demand for the healthy alternative' food has left many manufacturers in a quandary. How do you reduce the level of fat or sugar in a food without adversely affecting the sensory properties, in particular the taste and flavour, and spoiling the consumers' enjoyment of the food? As consumers, we may not expect the 'healthy' alternative to taste the same as the standard recipe, and few (if we are honest) may expect it to taste better.

\section{The sensory experience}

There is a good deal of evidence that the sensory characteristics of food, in particular the taste and flavour, have a very specific effect on the consumers' food choice. In many ways the sensory attributes could be seen as a key area in which food manufacturers can differentiate their products.

The accessibility and choice of food in our society has never been as great as it is today. As we walk around our local food store we are bombarded with product information, tempting us to try this or that particular brand in favour of another. In-store tastings are commonplace, whether promoting new products or suggesting different methods of preparation and use. One major UK retailer now offers shoppers the opportunity to 'try before you buy' and will open almost any ready-to-eat product on demand. They will even chill the wine for you!

It seems that from the enticing aroma of freshly-baked bread, through to the Bavarian waltz music resounding in the wine aisles, the assault on our senses as we shop is unremitting. We are invariably drawn to interesting packaging designs, and the promises of smoother, crunchier, tastier products.

When we finally consume the food and experience the sensory characteristics (consciously or subconsciously), we are then able to make a decision on whether or not we like the food. The sensory characteristics, (the appearance, aroma, taste and texture) of a food will influence this decision to a greater or lesser extent.

If food producers can optimize the perceived sensory attributes of a product, this will help to increase its perceived value among consumers. We should not forget, for example, that a consumer may make up to fifty cups of coffee from one jar; that means fifty occasions on which to experience the sensory characteristics.

\section{Taste and flavour: the early years}

From an early age, our behaviour towards foods seems to be strongly influenced by the effects of taste and flavour. An example of this can be seen in a study of the facial expressions of young babies (Steiner, 1979). When given solutions of sweet and bitter compounds, the infant reacted in very different ways. Sweet tastes elicited a facial acceptance response, i.e. large eyes and retraction of mouth, resembling a smile. Bitter tastes gave a very different response, with tight closing of the eyes, gaping mouth and sudden turn of the head.

These instinctive reactions of young babies are indicative of a basic survival instinct in all of us. Put simply, it may be seen as the need for nutrition, hence the acceptance of the sweet solution $v$. the rejection of the possible harmful and poisonous substance, the bitter solution.

Some of these early pre-dispositions tend to remain with us throughout life, as we see from adult food cravings that are characterized by sweet tastes and pleasant smells. Some other food preferences, however, demonstrate a degree of acquired liking. An example of this is the liking for 'bitter'tasting lagers or hot spicy foods; tastes which as young infants we would instantly reject. 
The fact that we are able to acquire liking for different types of foods is an indication that taste and flavour characteristics, along with other sensory attributes, are influenced and moulded by other factors, such as our attitudes, beliefs and expectations.

\section{Consumer expectations}

Recent studies of consumer expectations (Cadotte et al. 1987; Cardello \& Sawyer, 1992) have highlighted the strong links between what consumers expect from a product and their resulting perception of liking. The effect of consumers comparing their expectations of sensory properties with those actually delivered by the product is of particular interest in the current context.

Does the food look anything like the picture on the box? Does it perform in the way you were led to believe it would? Is it really as 'tasty' as claimed in the advertisement? This is an important area of which manufacturers should be aware. If expectations are to be built up around a product and yet not fulfilled, it could cost them dearly.

\section{Consumer attitudes and beliefs}

Most people can say immediately if they do or do not like a particular food; however, it is not always as easy for them to describe the reasons why they like or dislike it. When prompted, consumers will often mention the taste and flavour as being a major factor in their preference decision, but do they always mean what they say?

In a study involving husbands and wives (Schafer, 1978), it was found that husbands rated taste, followed by nutrition, as the most important determinant of food choice. Conversely, the most important factor for wives was nutrition, followed by taste (Schafer, 1978). If we were to re-run this study today, perhaps we may expect food safety to come near the top of the list.

As we consume a food, the taste and flavour, along with the other sensory characteristics, are subject to both conscious and subconscious processing by the brain. There is, however, an element of 'noise' present during this process that is generated by other influences pertaining to the food itself: our personal beliefs and our economic and social position. Factors such as these are known to affect our eventual acceptance or rejection of the food.

Many models such as that shown in Fig. 1 have been developed to illustrate the different factors influencing consumers' food choice. Such models, however, are often unable to quantify the individual importance of each factor. It would appear that some foods are chosen after detailed processing and others are not. Sensory properties such as taste and flavour are probably more important for the latter products.

\section{Product optimization}

Understanding why consumers prefer some foods to others is of the utmost importance to food producers and suppliers in the 'real life' situations of marketing, advertising and new product development.

The problem of questioning consumers about the sensory characteristics of foods can prove to be counter-productive, due to the many other factors that are involved in the decision-making process. A clear example of this can be seen

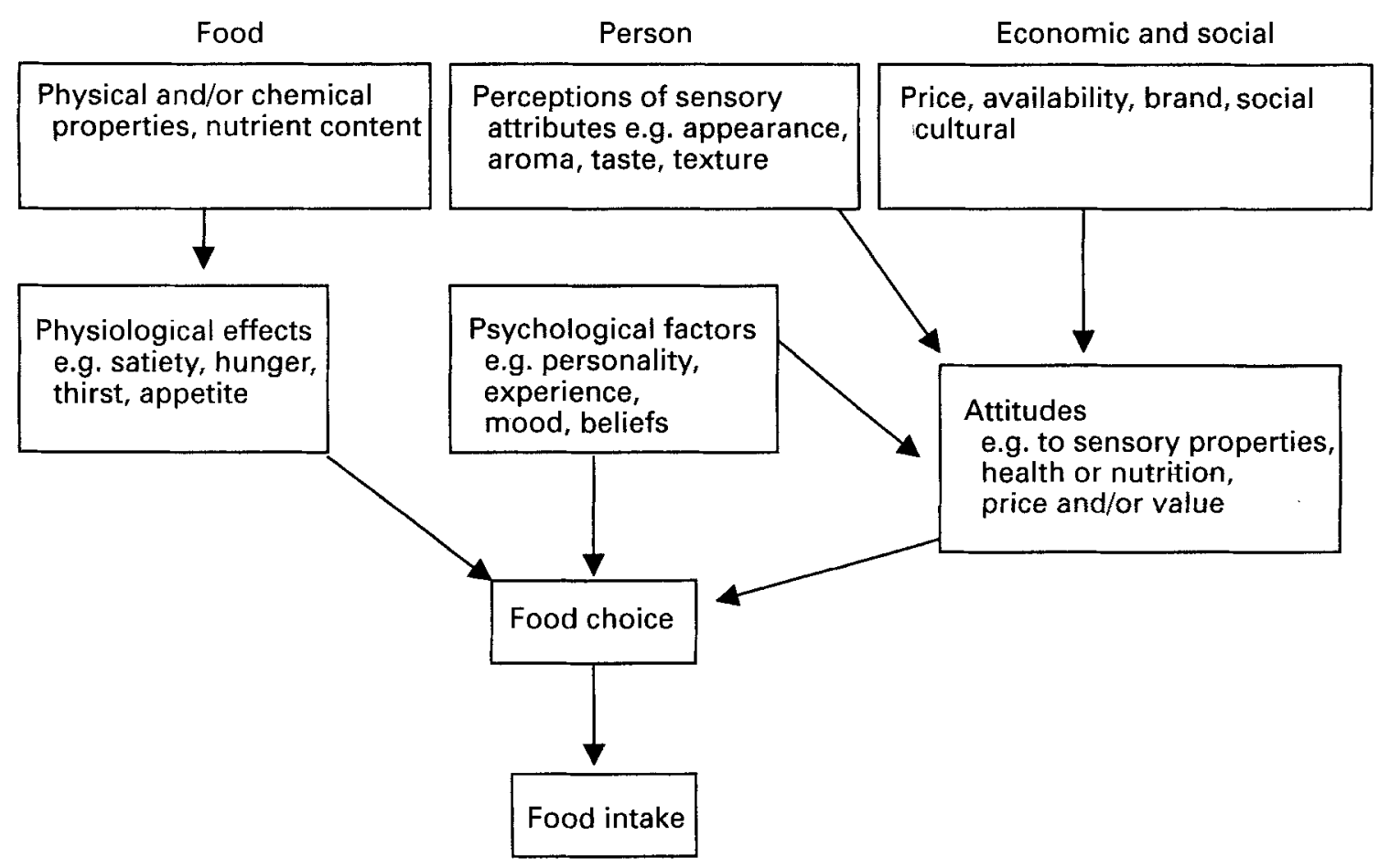

Fig. 1. Diagrammatic representation of consumer influences. (From Shepherd, 1985.) 
in the testing of coffee, where a consumer may say that a coffee should be less bitter but is not really sure what bitterness is. Could they be confusing it with acidity? Similarly, some people may think that they should be consuming less sugar in their diet, and as a result say that a product is too sweet, when in actual fact they like the taste.

Under normal everyday circumstances, a consumer would not have the need or the inclination to analyse the sensory characteristics of their food, at least as long as the product was not at odds with their expectations of sensory quality. They simply know what they like and what they dislike.

The conjecture concerning the usefulness of sensory information provided by consumers, together with the fact that it doesn't always correlate well with that of a trained sensory panel, led to the exploration of other methods of analysing consumer preferences. One such method is known as preference mapping (Carroll \& Chang, 1970; Nute et al. 1989). Preference mapping allows us to explain differences in consumer liking without asking consumers complex sensory-attribute questions.

\section{Preference mapping: the technique}

Preference mapping is a generic term involving a collection of techniques used to describe the relationship between consumer acceptance and sensory data.

The use of other consumer-research techniques such as paired preference (Rothman, 1972) and hedonic scaling (Williams, 1972), while generally thought to give a good measure of relative acceptance or the degree of product preference, can suffer from several disadvantages:

consumers often lack the ability to describe products using objective sensory terminology;

attribute scales can be used incorrectly by consumers; paired-preference data can often be difficult to analyse.

\section{Aims of preference mapping}

Preference-mapping techniques attempt to overcome the disadvantages of some other forms of analysis. One of its main uses is to describe how consumer acceptance may be influenced by the sensory characteristics of the product. It is particularly useful in product-development studies where altering a particular sensory attribute may affect consumer preference.

The preference mapping technique is basically simple. A panel of trained assessors is used to describe and quantify the sensory characteristics of a range of products. These products would be of a similar type, e.g. brands of coffee or vanilla ice cream. The number of products tested is usually large (more than six) and the proper training of the sensory panel is essential, as they need to be in accordance with each other in their understanding and scoring of each product attribute.

The data is collected from consumers by asking them to assess the same range of products hedonically. This is usually done through acceptance rating, or rank preference.

Data is analysed using statistical regression of the consumer acceptance data against the products' sensory scores. The main factors (sensory characteristics), which describe variability among the products provide the space on which consumer preference is then mapped.

\section{A case study}

The following case study involves the preference mapping of a range of rich fruit cake products. It explains how the methodology was put into practice, highlighting a major use of this technique in product development.

Ten products were evaluated in the test, and included the commissioning clients' current product and a new development sample, alongside other branded and retailer own label cakes. For the purposes of confidentiality, the actual product names have not been given.

First, a trained sensory panel identified, described and quantified the sensory characteristics of the product range. Consumer trials were then conducted using pre-recruited respondents who fulfilled the criteria required for the test, reflecting in particular their current purchase patterns regarding rich fruit cakes. The respondents attended tasting sessions at local venues, where they were briefed about the nature of the test, and asked to rate the acceptability of each of the ten samples on a nine-point hedonic scale. Each respondent reviewed the products in a balanced and unique order (Macfie et al. 1989). In total, 150 respondents took part in the test. Principal component analysis (Piggott \& Sharman, 1986) was carried out on the sensory data in order to identify the key differentiating sensory characteristics of the products. Preference mapping was then carried out by correlating the total sample mean scores onto the product co-ordinates. This produces what is known as a vector model (Carroll, 1972), which in this case is in the direction of the top left quadrant of the map (Fig. 2).

The analysis revealed that $48 \%$ of the respondents had similar preferences and these were fitted onto the map using the vector model. This implies that there is a direct relationship between preference and one or more attributes. The approximate acceptance ranking can be recovered from the projection shown in Fig. 2 by dropping perpendiculars from each product onto the vector line.

The direction of preference to the top left quadrant of the map (Fig. 2) therefore indicates that the most acceptable product is the own label $\mathrm{B}$, followed by the currentlyavailable product (S) and the new development sample (N).

By relating the respondents position to the known sensory dimensions of the product it is possible to infer which characteristics are determining preference. Thus, the majority of respondents positioned to the top left of the preference map (Fig. 2) prefer cakes that are sweet, nutty, fruity and crumbly. It was found that an 'ideal point' model (Carroll, 1972) best explained a further $21 \%$ of the respondents. This 'ideal point' infers that some products will have too much of a characteristic and others too little. Thus, there exists for some subjects a point that is the ideal strength for that characteristic (or combination of characteristics), which is the 'ideal point' referred to on the map (Fig. 2).

The clear direction of the preference vector has revealed a good consensus of opinion among a large percentage of respondents in this study. There was a clear preference for the sweeter, crumbly-textured cakes containing more nuts and fruit. The manufacturer's current product and the new 


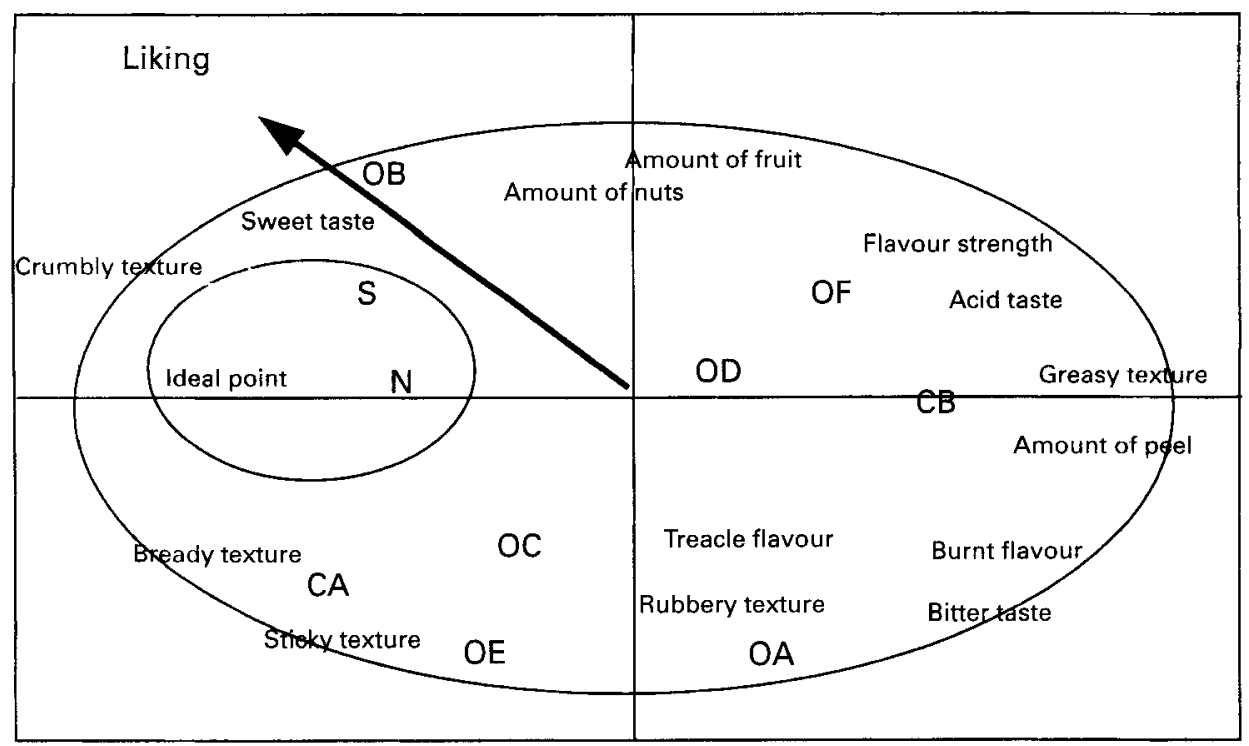

Fig. 2. Consumer preference map for fruit cake products, illustrating the main product attributes and the direction of overall preference. $S$, standard (current) product; N, new development sample; $O A-O F$, own label products; $C A, C B$, competitor samples.

development sample tended to fall into the 'ideal point' segment, indicating that there were upper and lower limits to acceptance based on their sensory characteristics.

This case study has shown that it is possible to separate respondents into particular segments, defined by similarities in response. These segments can then be further defined by information on socio-demographics, attitudes and behaviour, depending on what data has been collected. The major benefits of this type of test are that they allow the interpretation of consumer choice without bias.

Collecting consumer test data is a relatively easy procedure; however, it is the quality of the information that is important. Concentrating on hedonic and behavioural issues tends to enhance the data by minimizing bias and reducing the problems of misunderstanding and interpretation of attributes. Using a trained sensory panel is a very useful tool for revealing product differences. There is no doubt that problem solving can be made far easier through the use of such techniques, the results of which are far more actionable than some of the more standard methods of research.

\section{Conclusions}

We have seen that there is strong evidence that taste and flavour are important factors in consumer food choice, but that they may not be as far up the list as we anticipated. Many other factors are influential in modifying and moulding consumer behaviour in terms of their food preference decisions, as illustrated by Shepherd's (1985) model. The study by Steiner (1979) indicated the existence of innate sensory preferences for sweetness and dislike for bitterness and sourness. These anthropological origins are clear. We also know, however, that food preferences can be modified by continued consumption, e.g. the acquired preferences for spicy foods and bitter alcoholic drinks.

It would appear that some food choice decisions are arrived at only after complex conscious and subconscious processing by the brain. Other foods will not require such detailed cognitive processing, and it is thought that the sensory properties of these products could give a good prediction of consumer preference.

Product-optimization techniques such as preference mapping are very useful in defining which sensory characteristics may be driving consumer acceptance of particular foods. Asking consumers to rate or describe sensory properties of foods, however, can cloud our understanding of consumer acceptance patterns, but the collection of behavioural and attitudinal data will tend to enhance our interpretation.

More study is needed regarding the aspects of product expectations; in particular, the pattern of confirmation or disconfirmation of expected sensory properties. Finally, with the increasing use of sensory claims, both in advertising and on food packaging, it is important that the nature and quality of sensory information being passed to consumers is fully investigated and understood.

\section{References}

Cadotte ER, Woodruff RB \& Jenkins RL (1987) Expectations and norms in models of consumer satisfaction. Journal of Marketing Research 2, 305-314.

Cardello AV \& Sawyer FM (1992) Effects of disconfirmed expectations on food acceptability. Joumal of Sensory Studies 7 , 253-277.

Carroll JD (1972) Individual differences and multidimensional scaling. In Multidimensional Scaling: Theory and Applications in the Behavioural Sciences, vol. 1, pp. 105-155 [RN Shepard, AK Romney and SB Nerlove, editors]. New York, NY: Seminar Press.

Carroll JD \& Chang JJ (1970) Analysis of individual differences in multidimensional scaling via a $\mathrm{N}$-way generalization of 'Eckart-Young' decomposition. Psychometrika 35, 28-31.

Macfie HJH, Bratchell N, Greenhoff KG \& Vallis LV (1989) Designs to balance the effect of order of presentation and 
first-order carry-over effects in hall tests. Journal of Sensory Studies 4, 129-148.

Nute GR, Macfie HJH \& Greenhoff K (1989) Practical application of preference mapping. In Food Acceptability, pp. 377-386 [DMH Thomson, editor]. London: Elsevier.

Piggott JR \& Sharman K (1986) Methods to aid interpretation of multidimensional data, In Statistical Procedures in Food Research, pp. 181-232 [JR Piggott, editor]. London: Elsevier.

Rothman J (1972) Experimental designs and models. In Consumer Market Research Handbook, p. 35 [RM Worcester, editor]. Bath: Pitman Press.

Schafer RB (1978) Factors affecting food behaviour and the quality of husbands' and wives' diets. Journal of the American Dietetic Association 72, 138-143.

Shepherd R (1985) Dietary salt intake. Nutrition and Food Science 96, 10-11.

Steiner JE (1977) Facial expressions of the neonate infant indicating the hedonics of food related chemical stimuli. In Taste and Development: The Genesis of Sweet Preference. DHEW Publication no. NIH 77-1068, pp. 173-189 [JM Weiffenbach, editor], Washington, DC: US Government Publishing Office.

Williams JM (1972) Questionnaire design. In Consumer Market Research Handbook, pp. 84-86 [RM Worcester, editor]. Bath: Pitman Press. 